\title{
P2P Human-Resource Sharing and Its Redistribution Strategy of Stable Coin
}

\author{
Miki Saito *, Hisashi Hayashi *
}

\begin{abstract}
Workloads fluctuate in many small and medium-sized enterprises (SMEs). Managing human resources in these fluctuating conditions is difficult for many companies. During busy periods, workers struggle to complete all their work; however, during idle periods, they may not have sufficient work. The following are the two aims of this study: first to propose a new peer-to-peer (P2P) human-resource-sharing platform to balance the work hours of employees, and second to propose new reallocation strategies of stable coins called share $\mathrm{P}$ (sharing point). The first aim enables companies that are experiencing idle periods to offer their employees to other companies that are busy, by using an automated-negotiation technology. The second aim eliminates the share $\mathrm{P}$ bias that prevents smooth circulation of both share $\mathrm{P}$ and employees within the platform. The effectiveness of the new platform and new allocation strategy will be evaluated and verified via multi-agent simulation. Our simulation results show that idle/busy periods decrease approximately from one-third to one-half by using our P2P human-resource-sharing methods of employees. They can be further reduced to less than from one-fifth to one-seventh by using our new redistribution strategies of share $\mathrm{P}$.
\end{abstract}

Keywords: human-resource allocation, sharing economy, human-resource sharing, automated negotiation, peer-to-peer (P2P), multi-agent simulation, agent-based modeling

\section{Introduction}

In Japan, labor shortage is becoming increasingly serious. At the end of the year 2018, the Legal Committee of the House of Councilors passed an amendment to expand the acceptance of foreign workers. This new system was introduced in spring 2019 [1], and it might improve the labor-shortage situation. However, organizations must still consider the manner to appropriately utilize their human resources. In addition to the problem of chronic labor shortage in Japan, the SMEs Japan often experience significant workload fluctuations.

According to a survey by the Small and Medium Enterprise Agency, "busy periods response" and "short delivery response" are the main reasons for business practices leading to long

\footnotetext{
* Advanced Institute of Industrial Technology, Tokyo, Japan
} 
working hours for Japanese SMEs (which account for $99.7 \%$ of the total number of Japanese companies [2]). Particularly, as depicted in Figure 1, approximately $71 \%$ of SMEs experienced busy periods and the main reasons are "seasonal factors" and "delay in the response from business partners in busy periods", thereby resulting in long working hours of workers [3].

However, during idle periods, many workers are not utilized, thereby posing another problem. Indirect employment services, such as temporary staffing and outsourcing services, help companies adapt to variable workloads. However, considering long-term growth strategies, SME managers must avoid reducing direct employment more than required. Even in the conditions of changing workloads, a company must fully engage its labor force without relying considerably on indirect employment. This, in turn, helps develop the experience and skills of full-time employees.

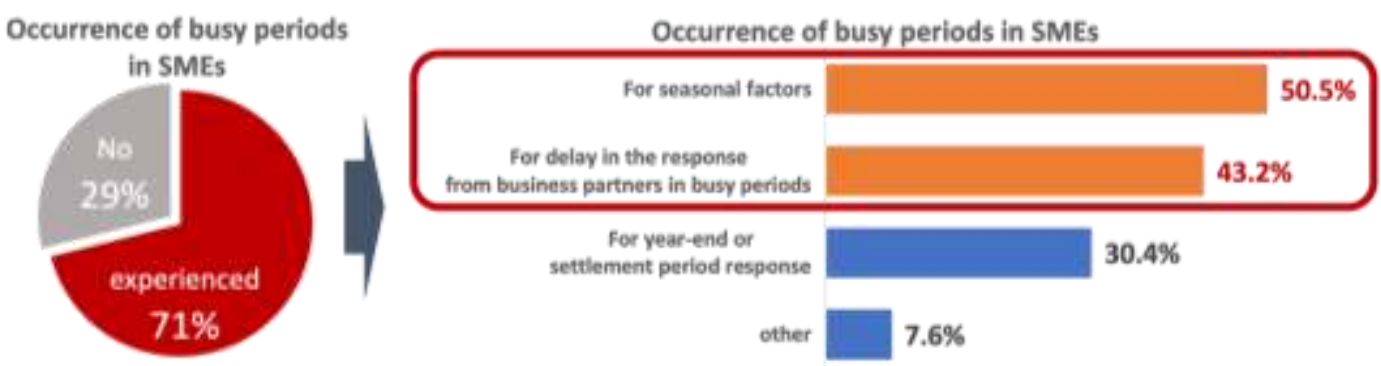

Figure 1: Ratio of busy periods and its reasons in SMEs

The business expansion due to the sharing economy [4], e.g., in companies such as Uber [5] and Airbnb [6], has occurred more rapidly than expected, including in Japan. The services in this sector range from vehicle- and residence-sharing to human-resource sharing, and they provide new values to customers in the societies in which they operate.

In our earlier version of this study, which was published in a conference proceeding [7], we explored ways for companies to level employee working hours without affecting sales. This might result in greater employment stability over time. In addition, we introduced a new intercompany, peer-to-peer (P2P), human-resource-sharing platform. Using the platform, companies during their idle periods can offer their employees to other companies that are busy by using an automated-negotiation technology [8]. In this extended version, we continue to explore ways for companies to level employee working hours on the basis of $\mathrm{P} 2 \mathrm{P}$ human-resource-sharing platforms; in addition, we propose new strategies that eliminate the share $\mathrm{P}$ bias, which prevents smooth circulation of both share $\mathrm{P}$ and employees within the platform.

In the previously proposed algorithms [9][10][11] based on human-resource allocation, information about all company agents needed to be centralized at one place to calculate the combinations of companies and transferred employees. However, in our P2P algorithm, when two companies negotiate for the transfer of employees, only the information about the two negotiating companies is required - an important consideration in terms of scalability and privacy. We evaluate and verify the effects of multiagent simulation.

The remainder of this paper is organized as follows. In Section 2, we review the literature on the domains of the sharing economy that employs $\mathrm{P} 2 \mathrm{P}$ platforms and human-resource allocation. The problem and context of this study are described in Section 3. Furthermore, in Section 4, we define the conceptual model for companies that share their employees. In Section 5, we define an automated-negotiation-based evaluation scenario for performing human-resource sharing. The simulation results are presented in Section 6. In Section 7, we analyze and discuss the 
simulation results. Finally, we conclude this paper in Section 8 by proposing ideas for future research.

\section{Related Work}

The term "sharing economy" was popularized by the media. Generally, it refers to the sharing of unused resources in return for a small fee or free of charge [12].

The sharing economy is related to the intersection of the following three trends [13]:

1) Circular economy [14]

2) Access economy

3) P2P economy

The P2P economy incorporates a network approach, similar to P2P file sharing innovation, and individuals share it offline. Conversely, users prepare for the exchange using an online platform, but ultimately exchange resources directly with each other in an offline manner [15].

In addition, the sharing economy involves the following two types of ownership with respect to the shared object (see Table 1):

1) The rental type involves the ownership of the shared object by the service provider.

2) The P2P type involves the ownership of the shared object by the user.

Table 1: Sharing type and ownership of objects

\begin{tabular}{|l|l|l|l|}
\hline Sharing type & Operator / form of service & Service contents & Ownership of shared objects \\
\hline 1) Rental type & Various rental services & $\begin{array}{l}\text { Rental (share) of various } \\
\text { objects }\end{array}$ & service provider \\
\hline 2) P2P type & e.g., Uber and Airbnb & $\begin{array}{l}\text { Brokerage share (cars } \\
\text { and facilities) }\end{array}$ & user (drivers and hosts) \\
\hline
\end{tabular}

In this study, as described previously, the following are the two categories of human-resource sharing:

1) Rental type (temporary staffing services)

2) P2P type: It is focused on the P2P-type inter-company human-resource sharing.

Unlike in the Rental type, in the P2P type, companies share their own employees, and the aim is to mediate the borrowing and lending of employees between companies. Resource allocation (e.g., resource sharing) is one of the following three aspects of resource management [16]:

1) Resource allocation

2) Resource monitoring

3) Resource discovery (resource provisioning)

For resource allocation, there are the following two kinds of algorithms [17]:

1) Centralized algorithms: A single manager agent calculates and allocates resources to its child agents.

2) Distributed algorithms: Multiple manager agents communicate and negotiate to share resources with other agents.

In the distributed algorithm of [18], agents adapt their resource allocation to a dynamic environment and gradually change the capacities of resources on the basis of the experience and predictions of an individual agent. However, in our study, each agent adjusts its own resource via automated negotiation and human-resource sharing. In automated negotiation, a group of 
agents reaches consensus by appealing to the rationality of one another [8]. Obviously, in resource allocation, automated negotiation is performed using distributed algorithms.

Furthermore, robust and adaptive optimization methods were proposed for performing human-resource planning in [9]; the methods were classified as centralized algorithms. In addition, multi-criteria human-resource-allocation methods for solving multistage combinatorial optimization problems using the multi-objective hybrid genetic algorithms in [10] were classified as centralized algorithms.

In the simulation scenario of [11], companies exchange employees by performing automated negotiation, which is considered a distributed algorithm. The needs of the companies (buyers) that are experiencing busy periods determine the price of the employees that are outsourced from companies (sellers) that are experiencing idle period. In addition, instead of targeting all their employees, companies classify them as either tradable or fixed. Both the companies in the busy periods and the companies in idle periods adopt a many-to-many auction format. Accordingly, when resource needs are high, the prices of workers also increase. However, in our study, we focus on the human-resource allocation of the distributed cooperative type and interchange structure. This $\mathrm{P} 2 \mathrm{P}$ human-resource-sharing platform enables the mutual exchange of employees by using a share $\mathrm{P}$, which has the property of stable coins, in such a way that the rate is always constant (1P per person), and the currency cannot be exchanged for real money. Therefore, it is insignificant for idle companies to lend their employees to other companies without borrowing workers from these companies during their own busy periods. Accordingly, companies can effectively reduce human-resource costs.

From the viewpoint of the circular economy, the share $\mathrm{P}$ that is temporarily stockpiled in idle periods by lending workers should be quickly used in busy periods for borrowing workers. However, while repeating this human-resource sharing, sometimes the bias of share $\mathrm{P}$ is found among some companies, thereby preventing the smooth circulation of share $\mathrm{P}$ within the platform. Accordingly, companies in idle periods cannot lend their employees to other busy companies that do not have sufficient share $\mathrm{P}$, thereby preventing the circulation of workers. The "redistribution strategy of share $\mathrm{P}$ " is one of the methodologies to resolve this bias, and it might improve the performance of the entire sharing platform.

\section{Problem and Issue Setting}

As stated in the abstract, Japan has a serious problem of labor shortage, and many SMEs experience significant workload fluctuations. Consequently, these companies struggle to maintain or increase their workforce. Starting from the spring of 2019, it is possible that nonJapanese citizens add to the labor force because of the Immigration Refugee Law amendment. However, human-resource allocation is still a significant problem for all the companies.

We aim to balance the working hours allocated to employees and lower the absolute values of "busyness and idleness." Accordingly, we propose an inter-company P2P human-resourcesharing platform using which companies in idle periods interact with companies in busy periods via automated negotiation, as depicted in Figure 2. We evaluated and verified the effect using a multiagent simulator implemented in NetLogo 6.1.1. 


\section{Concept Model 1: P2P Human-Resource Sharing}

Here, we describe the conceptual model of an inter-company P2P human-resource-sharing platform (see Figure 2). The platform adjusts the number of workers required by each company by exchanging their employees between companies via one-to-one automated negotiations.

In the following subsections, we introduce the concept of "agent, states, and resources," "factors affecting agents," "behavior model," and "internal model."

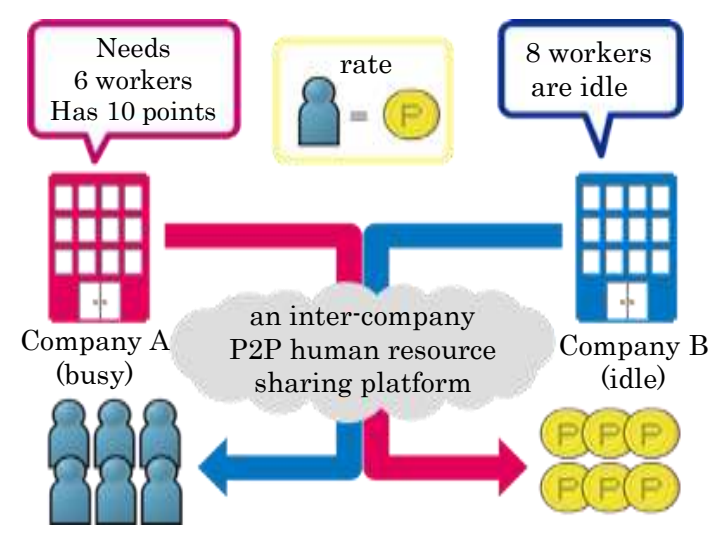

Figure 2: Negotiation diagram of an inter-company $\mathrm{P} 2 \mathrm{P}$ human-resource-sharing platform (1)

\subsection{Agent, states, and resources}

The only agent type is the "company agent," whose goal is to ensure that employees have adequate work and minimal overwork and to minimize the absolute value of the following:

(total working hours per day $\left.\left(\alpha_{c t}\right)\right)$ - (standard working hours per day)

$=\left(\right.$ overtime/idle working hours per day $\left.\left(\beta_{c t}\right)\right)$

To offset the "overtime/idle working hours per day $\left(\beta_{c t}\right)$," the company agents share their employees with other companies.

The company agents are classified into the following three states: "busy period," "idle period," and "normal period". More details are provided in Section 4.2. A centralized mediator agent is not required, as negotiations are conducted in a peer-to-peer manner. Notably, employees and share $\mathrm{P}$ are defined as the resources of company agents.

\subsection{Factors affecting agents}

External factors (environments) that affect corporate agents include the "total working hours per day $\left(\alpha_{c t}\right)$ " for the work ordered by clients, and these hours vary from day to day. The agents from the companies that are experiencing "busy periods" and "idle periods," negotiate and exchange.

"share P" and employees, to offset the "working hours of shortage or overage per day $\left(\beta_{c t}\right)$ " of that day, as depicted in Figure 3.

The agent variable "status" is determined using the "overtime/idle working hours per day $\left(\beta_{c t}\right)$," which is determined using the "total working hours per day $\left(\alpha_{c t}\right)$," which changes every day. The determination of "status" is performed as follows: 
if $\left(\beta_{c t} \geq \gamma\right)$, status = "busy period,"

if $\left(\beta_{c t} \leq-\gamma\right)$, status $=$ "idle period,"

if $\left(\left|\beta_{c t}\right|<\gamma\right)$, status = "normal period."

$\gamma=$ Prescribed working hours (fixed at $8 \mathrm{~h}$ per person)

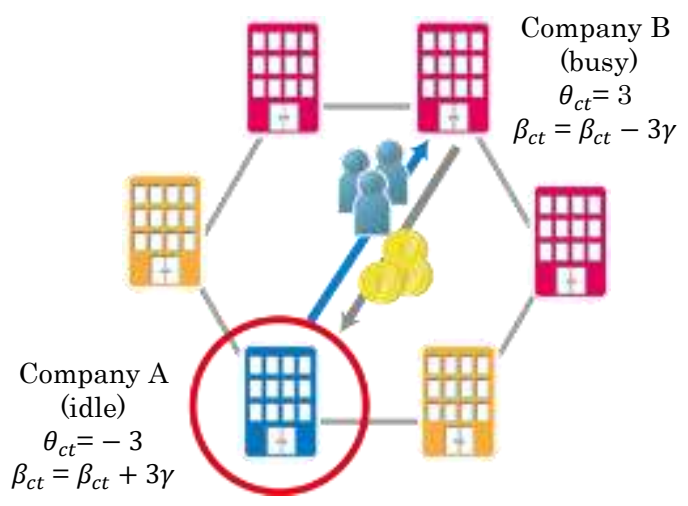

Figure 3: Negotiation diagram of an inter-company

P2P human-resource-sharing platform (2)

\subsection{Behavior model}

The main action of the company agent is to daily repeat Steps 1) -4) (ticks):

First, determine $\alpha_{c t}$ for that day.

1) If the status is idle (or busy), choose another company agent whose status is the opposite; subsequently, negotiate and then send (receive) employees if possible.

2) Complete the $\alpha_{c t}$ of the day, and do overtime work if necessary.

3) Plan to carry over any unfinished work to the next day.

4) Increase one tick (end the day).

\subsection{Internal model}

The variable $\beta_{c t}$ denotes the overtime/idle working hours per day (initial value 0 ). It is calculated using a formula according to which the (overtime/idle working hours per day) are equal to $\left(\left(\alpha_{c t}\right)\right.$ plus the carry-over working hours from the previous day) minus (the total working hours of all the employees including overtime hours and the work time of shared employees). The formula is mathematically presented as follows:

$\beta_{\mathrm{ct}}=\left[\alpha_{\mathrm{t}}+\max \left(0, \beta_{\mathrm{ct}-1}\right)\right]-\left[\mathrm{n}\left(\gamma+\delta_{\mathrm{ct}}\right)+\theta_{\mathrm{ct}} \gamma\right]$

$\alpha_{c t}=$ total working hours per day (fluctuates daily)

$\max \left(0, \beta_{c(t-1)}\right)=$ carry-over working hours from the previous day

$n_{c}=$ the number of employees (fixed)

$\delta_{c t}=$ overtime working hours per person (maximum: $30 \mathrm{~min}$; calculated after share negotiation; initial value is 0 )

$\theta_{c t}=$ the number of shared employees (determined during negotiation; initial value is 0 ; value is positive if borrowing employees from other companies and negative if lending employees to other companies)

$\gamma=$ Prescribed working hours (fixed at $8 \mathrm{~h}$ per person) 
Notably, $\theta_{c t}$ is determined during the negotiation between an idle-company agent and a busy-company agent and is set to the minimum value of the following: the number of idle employees of the idle company, the number of extra workers required by the busy company, or the amount of share $\mathrm{P}$ owned by the busy company.

In addition, $\delta_{c t}$ is obtained during the overtime-calculation process of the agent of the company in a busy or normal period. In the case of an idle period, $\delta_{c t}=0$. If the numerical value of the following formula is less than $0.5(30 \mathrm{~min})$, the value of $\delta_{c t}$ is as follows:

$$
\delta_{c t}=\frac{\alpha_{t}+\max \left(0, \beta_{c(t-1)}\right)-\left(n_{c} \gamma+\theta_{c t} \gamma\right)}{n_{c}}
$$

If the numerical value of the aforementioned formula is greater than or equal to 0.5 (30 $\mathrm{min}$ ), then $\delta_{c t}=0.5$, which is the upper limit of overtime. The portion that exceeds $30 \mathrm{~min}$ is carried over to the next day and processed while calculating $\beta_{(t+1)}$ for that day.

Next, we consider annual, monthly, and weekly working hours, such that one has the following:

annual- $\alpha=$ total working hours per year (fixed); calculated as $(n * \gamma * 240)$

monthly- $\alpha=$ total working hours per month (fluctuating)

weekly- $\alpha=$ total working hours per week (fluctuating)

$\mathcal{V}=$ cubic root of "variability rate" -1.00 (fixed)

The total working hours per month/week/day are varied by $\mathcal{V}$ and a random number and are calculated using the following equations 1) to 3), where random (x) denotes a function that represents a random number ranging from 0 to $\mathrm{x}$ in NetLogo.

monthly- $\alpha=$ annual $\alpha / 12+\operatorname{random}(\mathcal{V} *$ annual $\alpha / 6)-\mathcal{V} *$ annual- $\alpha / 12$

weekly- $\alpha=$ monthly- $\alpha / 4+\operatorname{random}(\mathcal{V} *$ monthly- $\alpha / 2)-\mathcal{V} *$ monthly- $\alpha / 4$

$\alpha_{t}=$ weekly- $\alpha / 5+\operatorname{random}(\mathcal{V} *$ weekly- $\alpha / 2.5)-\mathcal{V} \times$ weekly- $\alpha / 5$

$t=$ days $(240$ ticks per year, initial value 0$)$

Here, 1 week is equivalent to 5 days (Monday-Friday), 1 month to 4 weeks, and 1 year to 12 months (no holidays).

\section{Concept Model 2: Redistribution of Share $P$}

Here, we describe the redistribution strategy of share P. The underlying idea is that when share $\mathrm{P}$ does not smoothly circulate within the platform (see Figure 4-(a)), millionaire-company agents give some amount of share $\mathrm{P}$ to the 0pt-company agents that do not have any share $\mathrm{P}$ (see Figure 4-(b)), following which the 0pt-company agents can now borrow workers from other companies using the share $P$ received. (see Figure 4-(c)). 


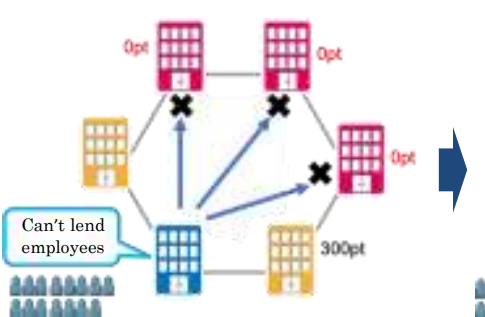

(a)

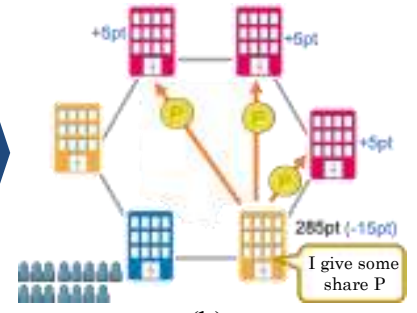

(b)

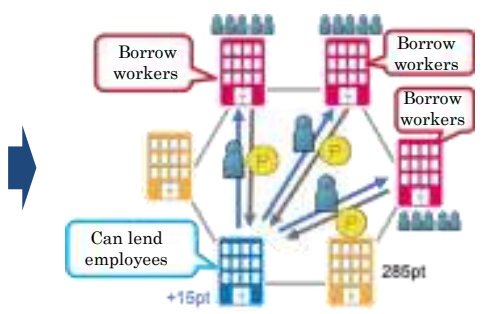

(c)

Figure 4: Redistribution strategy of sharing P

\subsection{Redistribution strategy of share $P$}

For the redistribution of share $\mathrm{P}$, two kinds of agents, namely, 0pt-company agent and millionaire-company agent, interact with each other. Notably, 0pt-company agents own no share $\mathrm{P}$ whether they are busy or idle. However, millionaire-company agents have more than sufficient share $\mathrm{P}$ and have the duty to give some amount of share $\mathrm{P}$ to 0pt-company agents. The ratio of the number of 0 pt-company agents to that of millionaire-company agents $(1: 1,2: 1$, and 3:1) is decided by the redistribution strategy of share P.

\subsection{Behavior model}

When the redistribution policy of share $\mathrm{P}$ is given, the following step, i.e., A), is added between usual Steps 3) and 4) (given in Section 4.3 Behavior model):

A) Examine whether there is a share $\mathrm{P}$ bias that 0pt-company agent exists within the platform, and if so, execute the strategy by following the redistribution policy of share P.

\subsection{Internal model}

Given the amount of share $\mathrm{P}$ (called redistributed $\mathrm{P}$ ) that is redistributed to each 0pt-company agent, the amount of share $\mathrm{P}$ (called duty $\mathrm{P}$ ) that each millionaire-company agent must pay is given as follows:

duty $\mathrm{P}=$ (number of 0 pt-company agents allocated to each millionaire) * redistributed $\mathrm{P}$.

Because the exchanges for share $\mathrm{P}$ are only integers, some share $\mathrm{P}$ is left after the division process. This share $\mathrm{P}$ will be cashback to a millionaire-company agent that paid tax and had the least amount of share $P$.

The adjustment of duty $\mathrm{P}$ is made for the following exceptional cases:

If a millionaire-company agent does not have the amount of duty $\mathrm{P}$, then the duty $\mathrm{P}$ of all the millionaire-company agents is reduced to the minimum share $\mathrm{P}$ of all millionaire-company agents.

In the end, even when redistributed $\mathrm{P}$ is set considerably high, if all the millionaire-company agents cannot pay for it, redistributed $\mathrm{P}$ is reduced. 


\section{Evaluation Criteria}

Here, we introduce three evaluation criteria. All of them are values for one year (240 ticks).

Criterion (1) "busyness and idleness per worker" represents the sum of the absolute value of $\beta_{c t}$ (the overtime/idle working hours per day) divided by $n_{c}$, i.e., the number of employees. The smaller, the better. It is given as

$$
\text { "Busyness and idleness per worker" }=\sum_{t=0}^{240}\left|\frac{\beta_{c t}}{n_{c}}\right|
$$

Criterion (2) "The number of borrowed persons per year" represents the total number of workers borrowed from other companies. The larger, the better. It is given as

$$
\text { "The number of borrowed workers per year" }=\sum_{t=0}^{240} \max \left(\theta_{\mathrm{ct}}, 0\right)
$$

Criterion (3) "The ratio of HR sharing" represents the total number of borrowed workers divided by the number of lent workers. The larger, the better. It is given as

$$
\text { "The number of lent workers per year" }=\sum_{t=0}^{240}\left|\min \left(\theta_{\mathrm{ct}}, 0\right)\right|
$$

"The ratio of borrowed to lent workers" = "\# of borrowed workers" / "\# of lent workers"

\section{Simulation Scenario}

Using the scenarios presented in this section, we analyze the behavior of the inter-company human-resource sharing in an artificial SME group of 17 companies. We conducted simulations for the following scenarios:

5 situation scenarios $x$

6 policy scenarios ( 3 types of share-partner selections +3 share P redistribution strategies)

\subsection{Situation scenario}

The situation scenario was analyzed using five different variability rates.

Situation (1): Variability rate of 1.00 (Total working hours per day do not change at all)

Situation (2): Variability rate of 1.25

Situation (3): Variability rate of 1.50

Situation (4): Variability rate of 1.75

Situation (5): Variability rate of 2.00 (Total working hours per day fluctuates more) 


\subsection{Policy scenario 1: Selection of share partner}

In addition to sharing $(0)$ in which company agents do not share any workers, we tested sharings (1) and (2), in both of which company agents share workers. In all the policies, including Policy (0), the occurrence of overtime is possible, up to 30 minutes per person per day. Furthermore, a share cycle event occurs once per day. All the company agents whose status is busy or idle can negotiate with idle (busy) company agents during one share cycle.

Policy (0): No sharing (default)

Employees complete the total required working hours by themselves.

Policy (1): Negotiate with one randomly selected company.

An agent from an idle (or busy) company randomly selects an agent from a company whose status is the opposite (busy or idle). Subsequently, the agents negotiate labor sharing, and $\theta_{c t}$ employees are exchanged with the share $\mathrm{P}$ of $\theta_{c t}$.

Policy (2): Negotiate with one randomly selected company with the maximum value of $\left|\beta_{c t}\right|$.

Each idle (or busy) company agent selects a company agent whose status is the opposite (busy or idle) and whose $\left|\beta_{\mathrm{ct}}\right|$ is the maximum. Subsequently, the agents negotiate labor sharing, and $\theta_{c t}$ employees are exchanged with the share $\mathrm{P}$ of $\theta_{c t}$.

\subsection{Policy scenario 2: redistribution strategies of share $\mathbf{P}$}

The redistribution strategy of share P represents "the number of 0pt-company agents allocated to a millionaire-company agent." The duty P paid by a millionaire-company agent is calculated using the number of 0 pt-company agents and redistributed $\mathrm{P}$ that each 0 pt-company agent takes.

Policy (1): 1:1 (\# of 0pt-company agents allocated to a millionaire-company agent = 1) The number of millionaire-company agents is defined as the number of 0pt-company agents. Each millionaire-company agent pays duty $\mathrm{P}$ that is equal to the amount of redistributed $\mathrm{P}$ that one $0 \mathrm{pt}$-company agent receives.

Policy (2): 2:1 (\# of Opt-company agents allocated to a millionaire-company agent = 2) The number of millionaire-company agents is defined as half (round up) the number of 0pt-company agents. Each millionaire-company agent pays the duty $\mathrm{P}$ that is equal to the amount of redistributed $\mathrm{P}$ that two 0pt-company agents receive.

Policy (3): 3:1 (\# of 0pt-company agents allocated to a millionaire-company agent =3) The number of millionaire-company agents is defined as one-third (round up) of the number of 0pt-company agents. Each millionaire-company agent pays the duty $\mathrm{P}$ that is equal to the amount of redistributed $\mathrm{P}$ that three 0pt-company agents receive.

\section{Simulation Results}

On the basis of 5 situation scenarios and 6 policy scenarios ( 3 types of share partners +3 types of redistributions for share $\mathrm{P}$ ), we tested 30 scenarios in total and ran simulation 100 times (random seed 0 - 99) for each scenario.

First, we evaluated our HR-sharing platform without the redistribution of share P. Second, we evaluated the effect of the redistribution strategies of share P. The two evaluation criteria 
used were (1) the total values for "busyness and idleness per person" for all the companies and (2) the total values for "borrowed persons per year" for all the companies. Because the total number of "borrowed persons" is equal to the total number of "lent persons," "the ratio of borrowed to lent persons/workers" of Criterion (3) for all the company agents is always 1.0.

The initial values of the company agents are listed in Table 2 .

Table 2: Initial values for company agents

\begin{tabular}{|c|c|r|r|}
\hline ID & $\mathbf{n}_{\mathbf{c}}$ & share P & Annual- $\alpha$ \\
\hline 0 & 50 & 50 & 96000 \\
\hline 1 & 50 & 50 & 96000 \\
\hline 2 & 20 & 20 & 38400 \\
\hline 3 & 20 & 20 & 38400 \\
\hline 4 & 80 & 80 & 153600 \\
\hline 5 & 80 & 80 & 153600 \\
\hline 6 & 30 & 30 & 57600 \\
\hline 7 & 30 & 30 & 57600 \\
\hline 8 & 50 & 50 & 96000 \\
\hline 9 & 50 & 50 & 96000 \\
\hline 10 & 20 & 20 & 38400 \\
\hline 11 & 20 & 20 & 38400 \\
\hline 12 & 80 & 80 & 153600 \\
\hline 13 & 80 & 80 & 153600 \\
\hline 14 & 30 & 30 & 57600 \\
\hline 15 & 30 & 30 & 57600 \\
\hline 16 & 300 & 300 & 576000 \\
\hline
\end{tabular}

$n_{c}=$ number of employees (fixed)

The initial point-of-sharing is equal to $n_{c}$.

(This point is changed from that in the earlier version of this paper [7])

annual $\alpha=$ total working hours per year (fixed) calculated as $\left(n_{c} * \gamma * 240\right)$;

$\gamma=$ standard working hours (fixed at $8 \mathrm{~h}$ per person)

\subsection{Policies of share-partner selection}

Here, three policy types of share-partner selection are evaluated via simulation. These results are different from our earlier results [7], as we used different initial input data.

a) Situation (1): Variability rate of 1.00

Employees can complete all their work by themselves. The total working hours do not fluctuate (see Figure 5-(a)), and, therefore, HR-sharing is not required in this case (see Figure 5-(b)). 

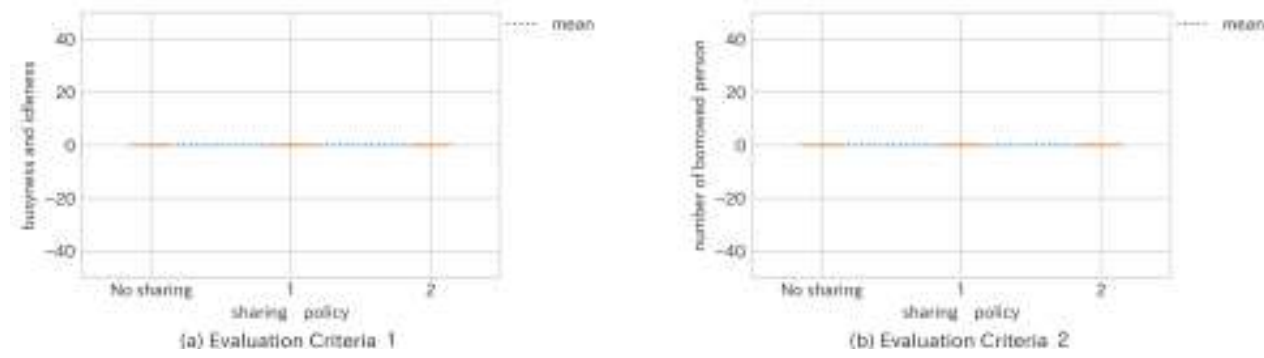

Figure 5: Graph for the variability rate of 1.00

\section{b) Situation (2): Variability rate of 1.25}

Evaluation criterion (1): Because the total working hours fluctuate depending on the day, the evaluation values accordingly vary. In sharing policies 1 and 2 , in both of which employees are shared among company agents, the average evaluation value is less than half than that in the case of no sharing, in which employees are not shared (see Figure 6-(a)).

Evaluation criterion (2): The average values of the persons borrowed per year are approximately $3300-3400$ in sharing policies 1 and 2 (see Figure 6-(b)).
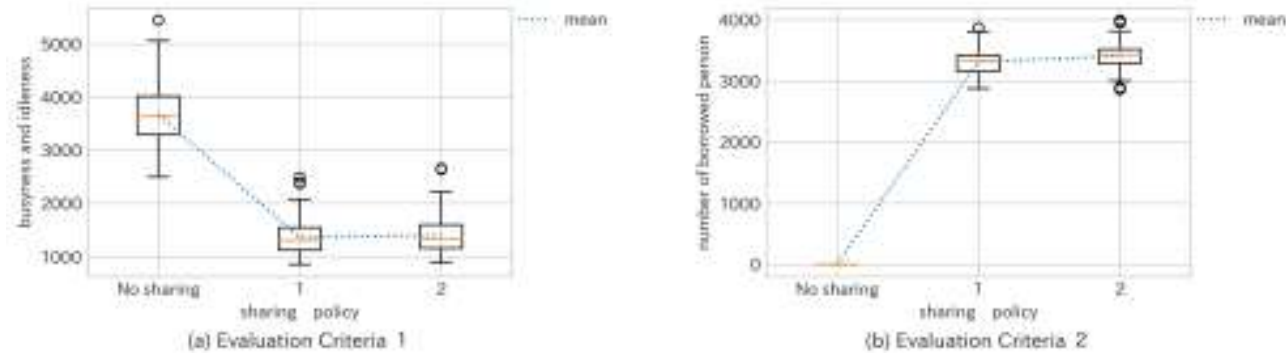

Figure 6: Graph for the variability rate of 1.25

c) Situation (3): Variability rate of 1.50

Evaluation criterion (1): We obtained almost the same result as that in Situation (2). In sharing policies 1 and 2, the average value of busyness and idleness is approximately $1 / 2$ lower than that in the case of no sharing (see Figure 7-(a)).

Evaluation criterion (2): The average values of the persons borrowed per year are approximately 5000 in sharing policies 1 and 2 (see Figure 7-(b)).
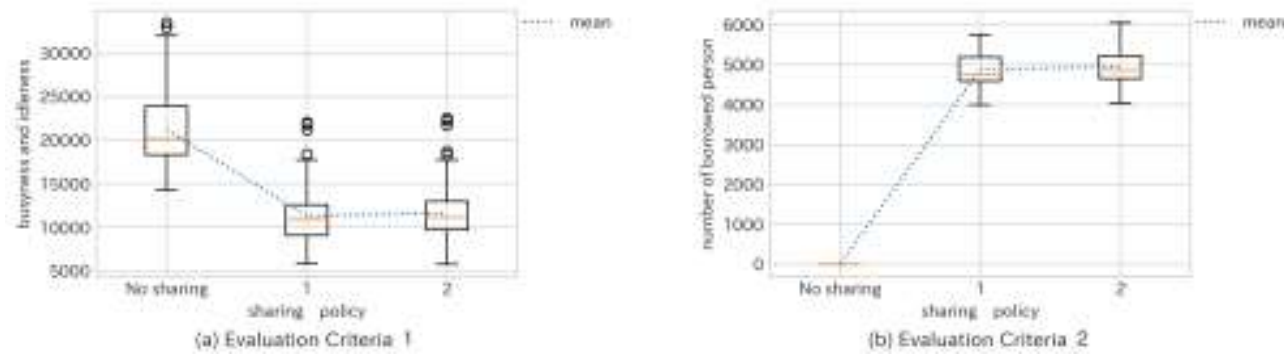

Figure 7: Graph for the variability rate of 1.50 


\section{e) Situation (4) / Variability rate of 1.75}

Evaluation criterion (1): The average value of busyness and idleness is approximately $1 / 3$ lower than that in the case of no sharing (see Figure 8-(a)). Compared with Situations (2) and (3), the decreasing rate has dropped from $1 / 2$ to $1 / 3$.

Evaluation criterion (2): The average values of the persons borrowed per year are less than 5000 in sharing policies 1 and 2 (see Figure 8-(b)).
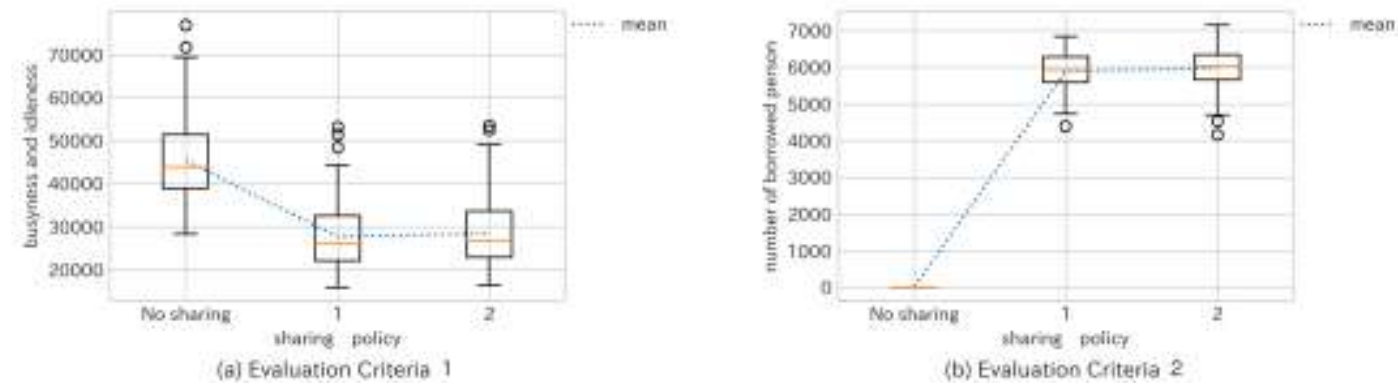

Figure 8:Graph for the variability rate of 1.75

\section{f) Situation (5) / Variability rate of 2.00}

Evaluation criterion (1): The average value of busyness and idleness is less than $1 / 3$ than that in the case of no sharing (see Figure 9-(a)). Compared with Situation (4), the decreasing rate $(1 / 3)$ is almost the same.

Evaluation criterion (2): The average values of the persons borrowed per year are more than 6000 in sharing policies 1 and 2 (see Figure 9-(b)).

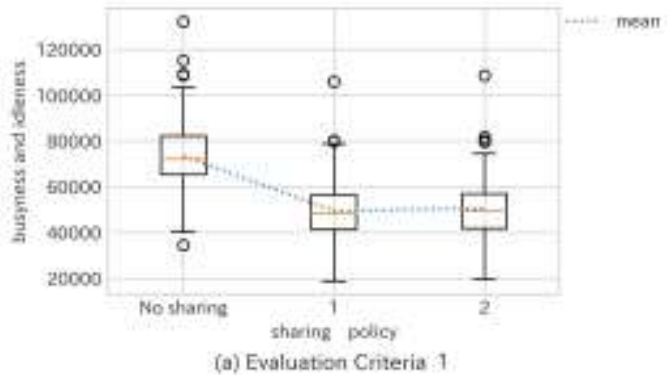

Figure 9: Graph for the variability rate of 2.00

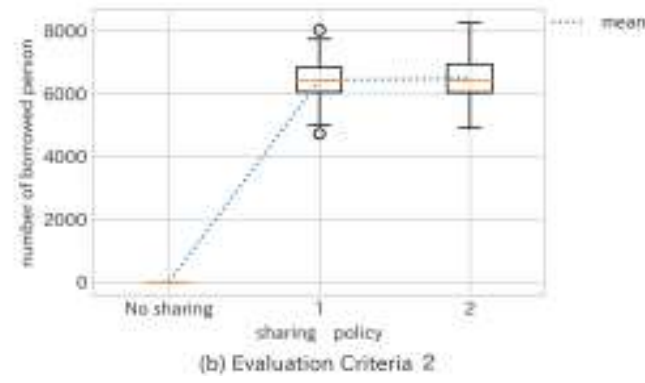

(b) Evaluation Criteria 2

According to the simulation results for evaluation criterion 1, it is asserted that Policies 1 and 2 - in which employees are shared - are more effective than "no sharing," in which employees are not shared. The average total values of "busyness and idleness per person per year" in all the companies significantly decreased during the implementation of Policies 1 and 2 . However, there is almost no difference in the average evaluation values between Policies 1 and 2 . These results suggest that the manner in which idle and busy company agents are matched is not 
important in our simulation scenarios. In addition, we observed that a single negotiation by each company agent per share cycle was sufficiently effective.

According to the simulation results for evaluation criterion 2, many workers shared in Policies 1 and 2 . The average values of the persons borrowed per year increase as the variability rate increases. However, when variability rates were 1.75 and 2.00 , the average values were almost the same.

\subsection{Policies of redistribution for share $P$}

Here, we evaluate the combination of two types of share-partner-selection policies ( 1 and 2$)$ and three kinds of redistribution strategies for share $\mathrm{P}(1: 1,2: 1$, and $3: 1)$ via simulation. We set the variability rate to 1.5 . The average values for evaluation criterion (1) are depicted in Figure 10. The horizontal line represents the amount of "redistributed P" that is redistributed to each 0ptcompany agent. The results of the six scenarios are similar to one another. As the amount of redistributed $\mathrm{P}$ increases, both busyness and idleness decrease. Therefore, the redistribution of share $\mathrm{P}$ is effective. However, the decreasing rates of busyness and idleness drop as the amount of redistributed $\mathrm{P}$ increases. While the redistributed $\mathrm{P}$ is between $1 \mathrm{pt}$ and $3 \mathrm{pt}$, the combination of sharing-partner-selection policy 1 and share $\mathrm{P}$ redistribution strategy 2-to-1 yields the best results, and, subsequently, sharing-partner-selection policy 2 yields good results.
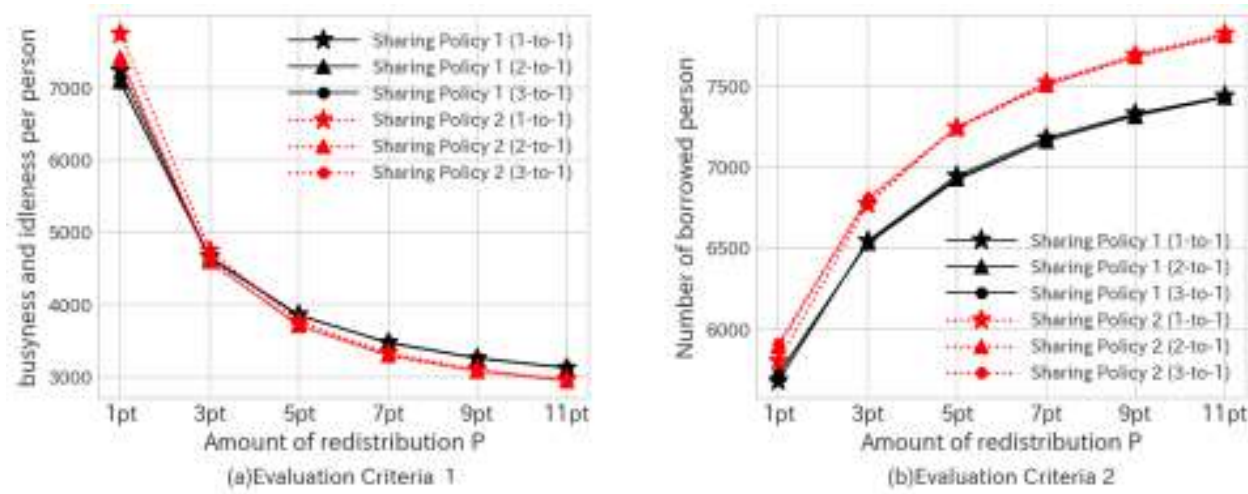

Figure 10: Comparison of busyness and idleness for different sharing policies

\section{Discussion and Further Experiments}

From the simulation results, significant effects of human-resource sharing have been confirmed in terms of reducing busyness and idleness and also reducing the number of borrowed workers. We also confirmed that the redistribution of share P further reduces the busyness and idleness to less than from one-fifth to one-seventh.

However, the following two question arises about the redistribution for share P:

Is it fair and effective for millionaire-company agents to give their share $\mathrm{P}$ to 0 pt-company agents? 
To answer this question, we further analyze each company agent in the following two cases. In these cases, we use the combination policy of share-partner-selection policy 2 and the share $\mathrm{P}$ redistribution policy of 3-to-1.

In Figure 11 and Figure 12, we depict the "busyness and idleness per person per year" for these two cases. The numbers of legends represent the ID numbers of company agents.

Case 1 (Multiple millionaire-company agents pay a lot, but the total duty amount is the smallest):

In this case, the total amount of duty P paid by multiple millionaire-company agents is the smallest when the amount of redistributed $\mathrm{P}$ is $5 \mathrm{pt}$. (see Figure 11). However, the busyness and idleness do not considerably change even if multiple millionaire-company agents pay significantly. Therefore, the amount of redistributed P must be kept low. In the future, we would like to find better solutions to this problem.
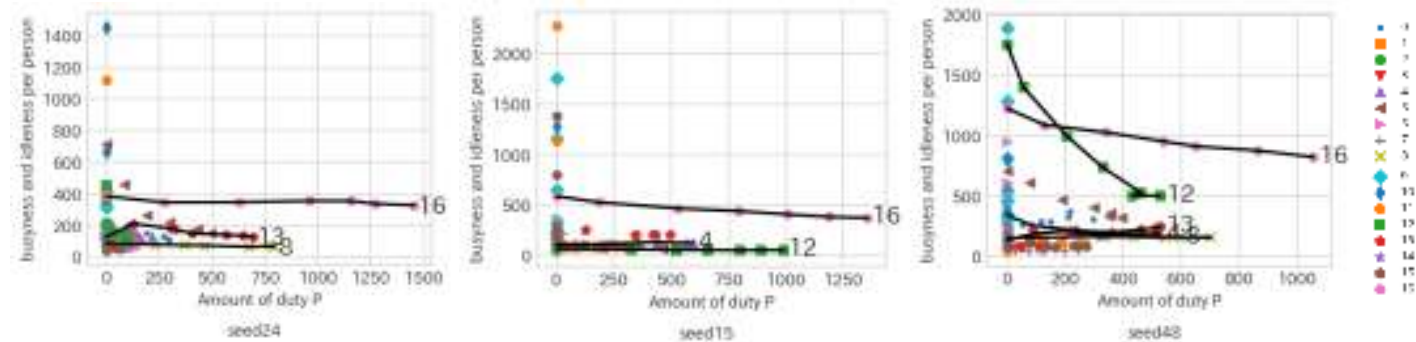

Figure 11: Comparison of busyness and idleness in case 1

\section{Case 2 (Only one millionaire-company agent pays a lot):}

When the amount of redistributed $\mathrm{P}$ is $11 \mathrm{pt}$, only the company agent 16 , which has the greatest number of employees $\left(n_{16}=300\right)$, pays considerably, and its busyness and idleness are not reduced (see Figure 12). However, it is not unfair for the other millionaire-company agents.
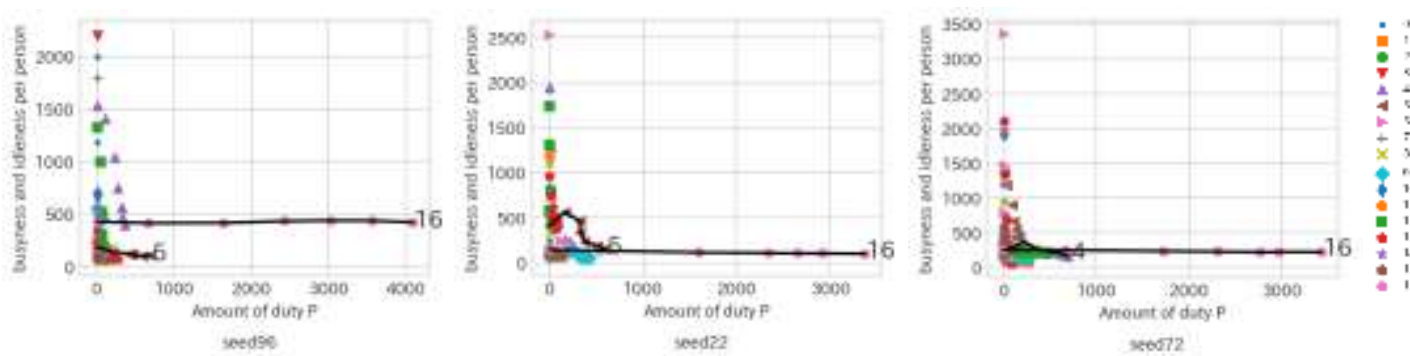

Figure 12: Comparison of busyness and idleness in case 2

\section{Other evaluations:}

Figure 13 and Figure 14 depict the average number of "borrowed persons per year," the number of "borrowed persons per year" for each company agent, and the "ratio of borrowed to lent persons" for these two cases, respectively. The numbers of legends represent the ID numbers of company agents. 
As a common trend, all the values for the "number of borrowed persons" are positive, thereby showing the effectiveness of this HR sharing. However, the "numbers of borrowed persons" of millionaire-company agents that pay considerable duty $\mathrm{P}$ does not significantly increase. The "ratio of borrowed to lent persons" is more than 0.5 , which is a satisfactory number, for most company agents. However, this ratio tends to decrease as the amount of duty $\mathrm{P}$ increases. Therefore, it is better to keep the amount of redistributed P low. In the future, we would like to find better solutions to these problems.

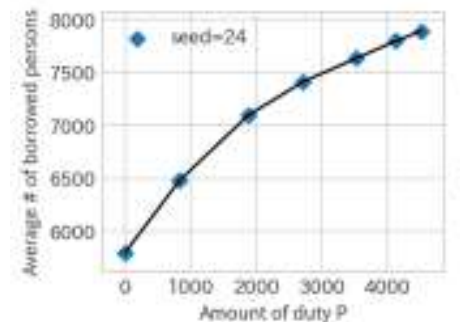

(a) Evaluation Criteria 2

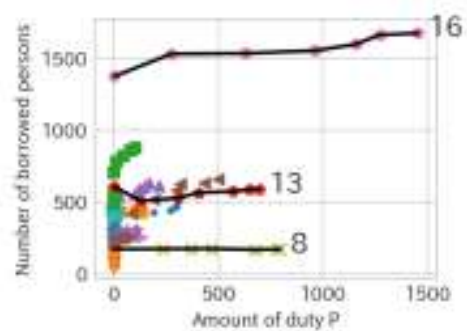

(b) Evaluation Criteria 2(Per agent

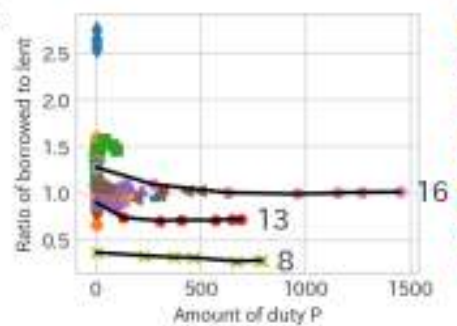

(c) Evaluation Criteria 3(Per agent)

Figure 13: Other evaluations in case 1 (seed24)

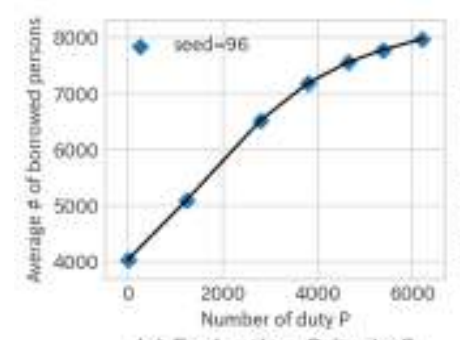

(a) Evaluation Criteria 2

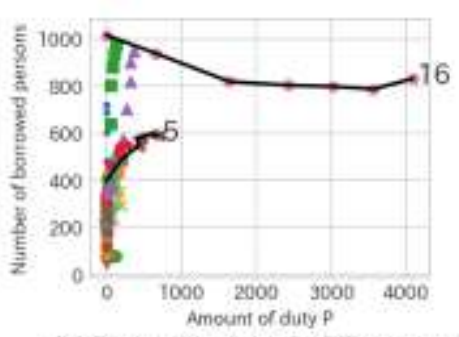

(b) Evaluation Criteria 2(Per agent)

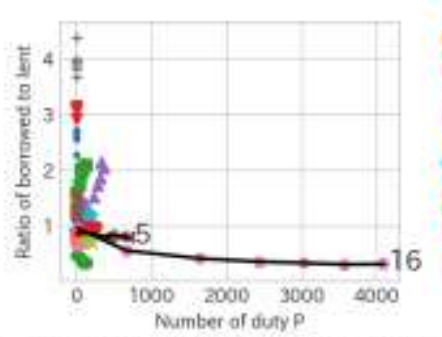

(c) Evaluation Criteria 3(Per agent)

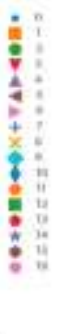

Figure 14: Other evaluations in case 2 (seed96)

\section{Conclusions}

In this study, we verified the effect of P2P human-resource sharing among SMEs that use a virtual currency (share P), via multi-agent simulation. We simulated Situation Scenarios (five types) $\times$ Policy scenario (three types of share partner selection + three types of redistribution of share P) 100 times for each scenario and evaluated each scenario on the basis of the total value of "busyness and idleness per person per year" and "borrowed persons" in all companies, and "the ratio of borrowed to lent persons". The idle/busy periods decreased approximately from one-third to one-half using our P2P human-resource-sharing methods. They could be further reduced to less than from one-fifth to one-seventh using our new redistribution strategies of share P. We confirmed that the "average" values of "busyness and idleness per person per year" became significantly lower because of human-resource sharing. In addition, we confirmed the effectiveness of HR sharing in terms of the persons borrowed per year and the ratio of borrowed 
to lent persons. However, some millionaire-company agents pay considerably high duty $\mathrm{P}$, and this problem will be the subject of our next study.

In addition, we would like to analyze more realistic scenarios and consider the following:

- Past experiences of HR sharing with specific companies.

- Adjustments and optimizes to eliminate unfairness.

- Both skill and other taxonomy associated with human resources [19].

- Parameter optimization using real data.

\section{References}

[1] The Mainichi Newspapers. "Japan ushers in major immigration policy change with revision to boost foreign workforce."

https://mainichi.jp/english/articles/20181208/p2a/00m/0na/013000c (visited on 2020)

[2] National Association of Trade Promotion for Small and Medium Enterprises. "2017 White Paper on Small and Medium Enterprises in Japan SME Life Cycle - Continuity in the Next Generation."

http://www.chusho.meti.go.jp/pamflet/hakusyo/H29/PDF/2017hakusho_eng.pdf(visited on 2020)

[3] National Association of Trade Promotion for Small and Medium Enterprises. "The web survey on business practices leading to long working hours of the SMEs"

https://www.meti.go.jp/press/2018/03/20190304006/20190304006.html (visited on 2020)

[4] A. Sundararajan. "The Sharing Economy: The End of Employment and the Rise of CrowdBased Capitalism.” The MIT Press.

[5] Uber Technologies Inc. "Get in the driver's seat and get paid." https://www.uber.com/ (visited on 2020)

[6] Airbnb Inc. "Vacation Rentals, Homes, Experiences \& Places - Airbnb." https://www.airbnb.com/ (visited on 2020)

[7] Miki Saito, Kenta Komine, Aiko Nakato, Isshin Sasaki, and Hisashi Hayashi. "Evaluating P2P human resource allocation strategies through multi-agent simulation." 7th International Conference on Smart Computing and Artificial Intelligence (SCAI), 8th International Congress on Advanced Applied Informatics (IIAI-AAI), pp. 506-510, 2019.

[8] S. Fatima, Sarit Kraus, Israel, and Michael Wooldridge. "Principles of Automated Negotiation." Cambridge University Press, London, 2014.

[9] L. Berk, D. Bertsimas, A. M. Weinstein, and J. Yan. "Prescriptive analytics for human resource planning in the professional services industry." European Journal of Operational Research 272(2) pp. 636-641, 2019. 
[10] C.-M. Lin and M. Gen. "Multi-criteria human resource allocation for solving multistage combinatorial optimization problems using multiobjective hybrid genetic algorithm." Journal of Expert Systems with Applications 34(4), pp. 2480-2490, 2008.

[11] S. S. Fatima and M. Wooldridge. "Adaptive Task and Resource Allocation in Multi-Agent Systems." AGENTS '01 Proceedings of the fifth international conference on Autonomous agents, pp. 537-544, 2001.

[12] H. Guyader and Laura Piscicelli. "Business model diversification in the sharing economy: The case of GoMore.” Journal of Cleaner Production 215, Elsevier Ltd, pp. 1059-1069, 2019.

[13] K. Frenken, "Political economies and environmental futures for the sharing economy." Journal of Philosophical Transactions A, The Royal Society. London, 2017.

[14] P. Lacy and J. Rutqvist. "Waste to Wealth: The Circular Economy Advantage." Palgrave Macmillan, 2015.

[15] T. Vaskelainen and L. Piscicelli. 2018. "Online and offline communities in the sharing economy.” Journal of Sustainability 10(8), 2018.

[16] F. Nzanywayingoma and Y. Yang. "Efficient resource management techniques in cloud computing environment: a review and discussion." International Journal of Computers and Applications 41(3), pp. 165-182, 2019.

[17] H. Hayashi. "Evaluating Task-Allocation Strategies for Emergency Repair in MAS." Transactions on Computational Collective Intelligence XXVIII, Lecture Notes in Computer Science 10780, pp. 253-274, 2018.

[18] She, Y., Leung, H.-F “An adaptive strategy for allocation of resources with gradually or abruptly changing capacities" International Conference on Tools with Artificial Intelligence, ICTAI 2,4669803, pp. 415-422, 2008 Proceedings.

[19] M. Arias, J. Munoz-Gama, and M. Sepulveda. "Towards a Taxonomy of Human Resource Allocation Criteria.” Business Process Management Workshops, pp. 475-483, Springer, 2018. 